\title{
Verzeichnis des Schrifttums
}

\section{Kommentare zum Personenstandsgesetz}

\section{PStG 1875}

Emig: Gesetz über die Beurkundung des Personenstandes und dieEheschließung, 1933

Fidler: Das Reichsgesetz über die Beurkundung des Personenstandes und die Eheschließung in der vom 1. Januar 1900 an geltenden Fassung usw., 1901

Hinschius-Boschan: Das Reichsgesetz über die Beurkundung des Personenstandes und die Eheschließung vom 6. Februar 1875, 4. Aufl., 1909

Philler: Das Gesetz über die Beurkundung des Personenstandes und die Eheschließung, 2. Aufl., 1900

Sartorius: Kommentar zum Personenstandsgesetz in der vom 1. Januar 1900 an geltenden Fassung usw., 1902

Sauer: Das deutsche Personenstandsgesetz in seiner neuen Fassung, 1925

Sicherer, v.: Das Reichsgesetz über die Beurkundung des Personenstandes usw., 1881

Stölzel-Spieler: Das Personenstandsgesetz in heutiger Gestalt usw., 4. Aufl., 1936

Weber: Das Reichsgesetz über die Beurkundung des Personenstandes usw., 1875

Wiest: Das Reichsgesetz über die Beurkundung des Personenstandes usw. in der vom 1. Januar 1900 ab geltenden Fassung, 2. Aufl., 1900

\section{PStG 1937}

Brandis-Massfeller: Das neue Personenstandsgesetz vom 3. November 1937 und Ausführungsvorschriften, 1938

Emig: Personenstandsgesetz mit der 1. AusfVO, der amtlichen Begründung und anderen einschlägigen Vorschriften, 2. Aufl., 1938

Massfeller: Das gesamte Personenstandsrecht, Frankfurt 1951

Peters: Personenstandsgesetz - Textausgabe mit Hinweisen, 3. Aufl., 1956

Schlempp: Das neue Personenstands- und Eherecht, 1939

Stölzel: Personenstandsgesetz nebst Ergänzungen und Erläuterungen, 5. Aufl., 1939

\section{PStG 1957}

Feneberg-Simader : Personenstandsgesetz - Handkommentar, 1958

Koehler-Massfeller : Personenstandsrecht, 1957.

\section{Dienstanweisung für die Standesbeamten und ihre Aufsichtsbehörden}

Koehler-Mäder: Dienstanweisung für die Standesbeamten und ihre Aufsichtsbehörden — Neufassung, 1958

Simader: Dienstanweisung für die Standesbeamten und ihre Aufsichtsbehörden, 2. Aufl., 1958 
Verzeichnis des Schrifttums

\section{Sonstiges Schrifttum}

Achilles-Greiff : Kommentar zum Bürgerlichen Gesetzbuch, 21. Aufl., 1958

Ahlborn: Nachträgliche Beurkundung von Geburten polnischer Kinder, StAZ 58,80 ;

Zur Behandlung vor dem Inkrafttreten des PStG 1937 eingetretener Legitimationen, StAZ 58, 108

Albers: Die Befreiung von der Beibringung des Ehefähigkeitszeugnisses, StAZ $54,145,170$;

Einzelfragen der Befreiung von der Beibringung des Ehefähigkeitszeugnisses, StAZ 51, 224; 52, 49

Arnold: Reform der Ehemündigkeit, StAZ 52, 246

Bach : Führung früherer Adelsbezeichnungen im Sudetenland und im Protektorat Böhmen und Mähren, StAZ 41, 121

Bachmann : Abgrenzung zwischen Lebend- und Totgeburten, StAZ 55, 118;

Behandlung von Angehörigen der amerikanischen Truppen und sonstigen unter die amerikanische Militärgerichtsbarkeit fallenden Personen auf dem Standesamt nach Eintritt der Souveränität der Bundesrepublik Deutschland, StAZ 55, 189;

Benachrichtigung der ausländischen Konsularbehörden im Inland über eingetretene Sterbefälle von Ausländern, StAZ 55, 240;

Berufsangabe der als Witwe oder als geschiedene Frau Verstorbenen im Sterbeeintrag, StAZ 53, 137;

Bescheinigung über eine Totgeburt zur Vorlage bei der Krankenkasse, StAZ 55, 143;

Beurkundung von unbekannten aus Trümmern geborgenen Leichen und von unbekannten Totgeburten, StAZ 53, 188;

Das neue Familienbuch in der standesamtlichen Praxis, StAZ 58, 154, 243, 295 ; 59,74 ;

Die Adoption deutscher Kinder durch Angehörige der Vereinigten Staaten von Amerika, StAZ 55, 36;

Die Bezeichnung des Familienstandes des Verstorbenen im Sterbeeintrag, StAZ 50, 162;

Ein deutsches Kind wurde zweimal adoptiert, einmal in Deutschland, einmal in den USA, StAZ 53, 236;

Fotokopie und Mikrokopie im Dienste der standesamtlichen Arbeit, StAZ 54, 15 ;

Inwieweit hat der Standesbeamte die ihm obliegende Pflicht zur Amtsverschwiegenheit bei Erteilung von Auskünften oder Rückgabe von Belegen aus den Heiratssammelakten einzuhalten? StAZ 58, 129;

Ist der Geburtseintrag eines ehelichen, aber nach dem Tode des Vaters geborenen Kindes änderungsbedürftig, wenn die Ehe der Kindeseltern erst nach erfolgter Geburtsbeurkundung für nichtig erklärt wird? StAZ 54, 116;

Kann der Standesbeamte auf die Erhebung gesetzlich vorgeschriebener standesamtlicher Gebühren ganz oder teilweise verzichten und macht ein ausgesprochener Verzicht den Standesbeamten der Gemeinde gegenüber schadensersatzpflichtig? StAZ 56, 97;

Tragisches Ableben, StAZ 57, 135;

Vaterschaftsanerkennungserklärung von Besatzungsangehörigen, StAZ 55, 22;

Vornamen in lateinischer Form und Vornamen mit Zusätzen, StAZ 53, 214

Baumann: Der strafrechtliche Schutz des Personenstandes, StAZ 58, 225

B a u m b ch-La u terbach : Zivilprozeßordnung, 25. Aufl., 1958

Baur: Anmerkung zum Beschluß des BGH vom 15. Dezember 1956, FamRZ 57,124 
Bechtold: Ist die Anderung der Eintragung „Dissident“ in ,konfessionslos“ zulässig? StAZ 56, 224;

Kann der Standesbeamte ein unter Zwang erfolgtes Aufgebot ablehnen? StAZ 52, 258

Becker: Die Berichtigung der Personenstandsbücher, StAZ 50, 40;

Nochmals: die Verfassungswidrigkeit des $\S 67$ des Personenstandsgesetzes, FamRZ 57, 40

Behr: Zum Inhalt der Sterbefallmitteilung, StAZ 59, 305

Beilicke: Der Standesbeamte im Verkehr mit dem Publikum, StAZ 57, 130

Beitzke: Adoptionen ins Ausland, StAZ 48, 54; Das Verhältnis von Justiz und Tätigkeit, 1957; Verwaltung im Bereich standesamtlicher

Die Eintragung der Adoption ins Standesregister, FamRZ 56, 172;

Die Nachprüfung der Adoption durch den Standesbeamten, StAZ 54, 217;

Erneute Adoption nach dem Tode der Adoptiveltern? StAZ 55, 3;

Familienrecht, 9. Aufl., 1960;

Internationale Zuständigkeit in Legitimationssachen, in: Festschrift für Herbert Kraus, 1954, S. 20;

Standesamt und freiwillige Gerichtsbarkeit, StAZ 59, 309;

Unwirksame Auslandsadoptionen, StAZ 53, 97;

Zur Anerkennung ausländischer Ehelichkeitsanfechtungen, StAZ 60, 89;

Zur Anerkennung ausländischer Ehescheidungsurteile, DRZ 46, 172;

Zur Anerkennung sowjetzonaler Eheurteile, FamRZ 56, 36;

Zur Eheschließung unter falschem Namen, StAZ 56, 65

Beitzke-Bachmann : Der Personenstand heimatloser Ausländer in Deutschland, 1952

Bergmann : Änderungen des Personenstandes, StAZ 31, 9;

Standesamt und Auslandsrecht, 3. Aufl., 1942;

Zur Einbenennung gemäB $§ 1706$ BGB, wenn ein Beteiligter Ausländer ist, StAZ 39, 167;

Zur Frage der Legitimation durch Sowjetangehörige, StAZ 32, 23

Berndt: ,Personenstandsgesetz“ in: von Bitter, Handwörterbuch der Preußischen Verwaltung, 2. Bd.

Bernstorff:, Graf von: Der Familienname in der deutschen Rechtsordnung, NJW 57, 1901

Beyer: Besonderheiten bei Eheschließungen mit Ausländern, StAZ 56, 37; Betrachtungen über die Befreiung von der Beibringung des ausländischen Ehefähigkeitszeugnisses, $\operatorname{StAZ} 57,29$

Bluhm : Die Eintragung der Adoption ins Standesregister, FamRZ 56, 269; Kann ich den Sohn meines Bruders heiraten? StAZ 57, 16

Böhler: Ist die handschriftliche Personenstandsbuchführung noch rationell und im Interesse der Lesbarkeit der Einträge noch vertretbar? StAZ 53, 68

Boehmer: Zur Rechtsstellung der durch nachfolgende Ehe legitimierten Kinder, insbes. zur Anwendbarkeit des $\$ 1593$ BGB, NJW 49, 52

Bonner Kommentar: Kommentar zum Bonner Grundgesetz, 1950

Bosch: Anmerkung zum Beschluß des OLG Nürnberg vom 28. Januar 1959, FamRZ 59, 116

Boschan: Deutsches zwischenstaatliches Personenstandsrecht - IV. Personenstandsfälle auf hoher See, StAZ 33, 168;

Personenstandsfälle auf deutschen Flüssen, Binnengewässern und Küstengewässern, StAZ 31, 260;

Zur Frage der Legitimation unehelicher deutscher Kinder durch Ausländer, insbesondere durch Russen, StAZ 30, 360 
Braga : Anmerkung zum Urteil des BGH vom 17. Oktober 1956, NJW 57, 1922;

Die Eingliederung des Saarlandes, FamRZ 57, 37;

$\mathrm{Mu} B$ die deutsche Frau ihr uneheliches Kind anerkennen, wenn sie den Vater französischer Staatsangehörigkeit heiratet? StAZ 52, 149

Brandis: Das neue deutsche Personenstandsgesetz, StAZ 37, 398;

Eintragung einer von einem Ausländer im Ausland erklärten Vaterschaftsanerkennung im deutschen Standesregister, StAZ 27, 35

Breidenbach : Das Vaterschaftsanerkenntnis, StAZ 58, 302;

Die Angabe des Wohnortes in den Einträgen der Personenstandsbücher und in den Personenstandsurkunden, StAZ 60, 212;

Die Mitwirkung der Meldeämter bei der Anforderung des Familienbuches, StAZ 60, 20;

Die Stellung des hauptamtlichen Standesbeamten gegenüber seiner Anstellungsbehörde, StAZ 57, 16;

,Wohnsitz", ,gewöhnlicher Aufenthalt" und „Wohnort", StAZ 59, 296

Brell : Die Sonderstellung des Standesamts I in Berlin, StAZ 50, 83;

Ubergang der Aufgaben des Hauptstandesamts in Hamburg auf das Standesamt $I$ in Berlin West, StAZ 52, 187

Brunn: Anerkennung von Sowjetzonenurteilen, NJW 56, 171

Buchheim: Anerkennung ausländischer Scheidungsurteile von Amts wegen? StAZ 52,234

Büchler, von: BGB § 1719 und EG. BGB Art. 22 Abs. 1, StAZ 31, 327

Bussjäger: Das Eheverbot des Ehebruchs liegt nicht vor, wenn gemäß § 52 Abs. 3 EheG auf Antrag des Beklagten nur die Mitschuld des Klägers im Urteil ausgesprochen ist, StAZ 54, 18

Christ: Wohnortangaben über die Eltern in den Spalten 4 und 5 des Familienbuches, StAZ 60, 182

Corves: Zur Nachprüfung der Adoption durch den Standesbeamten, SchlHA 56,97

Danner: Aushang des Aufgebots, Staatsangehörigkeit, Wartezeit, Auseinandersetzungszeugnis, Unterschriften der Verlobten im Heiratsbuch, Eintrag im Familienbuch, StAZ 58, 248;

Die Berufsbezeichnung eines Verstorbenen im Sterbebuch, StAZ 59, 192;

Die eidesstattliche Versicherung als Grundlage für die Eintragungen im Familienbuch, StAZ 60, 153;

Eheschließung mit einem Taubstummen, StAZ 60, 272;

Ist eine Vaterschaftsanerkennung im Geburtenbuch einzutragen, wenn sie nur von dem gesetzlichen Vertreter des minderjährigen Kindesvaters abgegeben wurde? StAZ 58, 221;

$\mathrm{MuB}$ der Familienstand der ledigen Kindesmutter beim Geburtenbucheintrag ihres Kindes mit eingetragen werden? StAZ 59, 76;

$\mathrm{Mu} B$ eine im Erstbuch vor Abschlu $B$ der Eintragung vorgenommene Berichtigung wörtlich in das Zweitbuch übernommen werden oder wird in das $Z$ weitbuch gleich der berichtigte Wortlaut eingetragen? StAZ 57, 81;

Vaterschaftsanerkennung und Geburtsurkunde für ein scheineheliches Kind, StAZ 58, 187;

Verlängert sich die einwöchige Anzeigefrist für eine Geburtsanmeldung auch dann, wenn der letzte Anmeldetag kein Sonntag, aber ein Feiertag ist? StAZ 59, 104;

Von welchem Alter an können Jugendliche einen Geburts- oder Sterbefall beim Standesamt anzeigen? StAZ 55, 244;

Wie sind Geburt und Tod eines neugeborenen Kindes, bei dem weder Lebendnoch Totgeburt nachgewiesen ist, standesamtlich zu behandeln? StAZ 57, 352 
David: Anerkennung eines unehelichen Kindes durch einen Sowjetrussen, StAZ 27, 24;

Kindesanerkennung durch einen Belgier, nachdem die Eheschließung stattgefunden hat, StAZ 27, 46

Delcker: Die Anerkennung unehelicher Kinder im Sinne des Art. 334 des luxemburgischen bürgerlichen Gesetzbuches, StAZ 37, 319;

Die Eintragung und deren Berichtigung im deutschen Standesregister bei bewußt unwahrer Vaterschaftsanerkennung deutscher unehelicher Kinder, StAZ 37, 82, 278

Denk: Die Änderung des Familiennamens der Frau durch Willenserklärungen, StAZ 60, 153;

Nachträgliche Geburtsbeurkundung nach § 41 PStG trotz Vorliegens eines auf Antrag der Eltern angelegten Familienbuches, StAZ 60, 332;

Vorsicht bei der Beurkundung von Geburten nach Auflösung der Ehe, StAZ 58, 79

Detert: Anerkennung der Vaterschaft und Legitimation unehelich geborener Kinder durch einen russischen Staatsangehörigen, StAZ 27, 318

Dippel: Aufhebung eines Adoptionsvertrages nach Eheschließung des Adoptierten, Geburtsbeurkundung seines ehelichen Kindes, StAZ 55, 89;

Geburt im Ausland, StAZ 50, 164;

Kann bei der Beurkundung eines totgeborenen Kindes ein Vorname eingetragen werden? StAZ 58, 137;

Standesamt und Standesamtsführung, 3. Aufl., 1958;

Ubersenden des Familienbuches nach Anforderung und im Gegensatz hierzu nach Eheschließung vor einem Standesbeamten, in dessen Bezirk der Mann nicht Wohnsitz oder gewöhnlichen Aufenthalt hat, StAZ 58, 215;

Vorschläge für die einheitliche Erledigung aller Arbeiten, die mit der Beurkundung einer Totgeburt im Zusammenhang stehen, StAZ 55, 63

Dürig: Die Verfassungswidrigkeit des $\S 67$ des Personenstandsgesetzes, FamRZ 55,337

Ehrensberger: Aufsicht und Rechtsweg im Personenstandswesen, StAZ 52, 145

Eichleiter: Ist das Kind einer deutschen Frau, die durch Urteil eines ausländischen Gerichts seit mehreren Jahren rechtskräftig geschieden ist, als unehelich zu beurkunden, so lange die Anerkennung des Urteils durch den Justizminister fehlt? StAZ 54, 94

Ellgaard: Beurkundung von Personenstandsfällen Deutscher im Ausland unter besonderer Berücksichtigung der dänischen Flüchtlingslager, § 41 PStG, $\S 265$ (1) DA, StAZ 59, 103;

Der LegitimationsfeststellungsbeschluB mit schwebender Wirkung, StAZ 51,275 ;

Einbenennung von Ausländern, StAZ 50, 87;

Hat die Frau das Recht zur Weiterführung des Ehenamens dadurch verwirkt, $\mathrm{da} B$ ihre Ehe vor Inkrafttreten des BGB für ungültig erklärt wurde? StAZ 58,25 ;

Ist „Kirsten“ ein männlicher oder ein weiblicher Vorname? StAZ 60, 248;

Legitimationsfeststellung bei Anwendung ausl. Rechts, StAZ 49, 217;

Namensführung des Kindes bei Adoption durch Dänen mit Wohnsitz in Dänemark, StAZ 59, 221;

Veränderte Ortsnamen und veränderte Standesamtsbezirke, StAZ 55, 237;

Wie ist das Vaterschaftsanerkenntnis eines heimatlosen Ausländers zu beurteilen, der die Bundesrepublik nach dem Anerkenntnis wieder verlassen hat? StAZ 57, 22

Elzemann: Testamentskartei beim Standesamt, StAZ 54, 185

Emmelmann: Zweifelsfragen im Legitimations-Feststellungsverfahren nach $\S 31$ PStG, StAZ 40, 280 
Erdsiek: Anmerkung zum Beschluß des LG Göttingen vom 2. März 1951, StAZ 52,158

Erman: Kurzkommentar zum Bürgerlichen Gesetzbuch, 2. Aufl,, 1958

Ermler: Von den besonderen Aufgaben des Standesamts I Berlin-West, StAZ 58,300

Ferid: Beischreibung der unehelichen Vaterschaft nach § 30 PStG auf Grund ausländischer Urteile? StAZ 53, 50;

Sind ausländische Verbote der Eheschließung mit Nichtstaatsangehörigen zu beachten? StAZ 54, 19

Ficker: Das Recht des bürgerlichen Namens, 1950;

Der Name der geschiedenen Ehefrau im deutschen internationalen Privatrecht, RabelsZ 50/51, 32;

Öffentliches Namensrecht, 1952;

Zur Namensführung der Ehefrau bei Ehen mit Auslandsberührung, StAZ 55, 214; Zum Statut der Einbenennung ( $\$ 1706$ II 2 BGB), AcP 151, 236

Fisch: Die Anerkennung außerehelicher Kinder durch den Vater nach schweizerischem Recht, StAZ 55, 255

Fischer : Erklärung über Hinzufügung des Mädchennamens zum Familiennamen des Mannes durch eine geschiedene Frau, StAZ 58, 249;

Fehlgeburt - Totgeburt, StAZ 59, 25;

Ist die Anerkennung des Urteils im Inland zur Geburtsbeurkundung eines nach der Ehescheidung geborenen Kindes erforderlich? StAZ 51, 212;

Mängel an der Vornamensgebung - Berichtigung oder behördliche Vornamensänderung? StAZ 55, 293;

Nachträgliche Beurkundung (Notbeurkundung) oder Beurkundung nach § 41 Abs. 1 PStG? StAZ 53, 66;

Namensuntersagung, nachdem die geschiedene Frau ihren Mädchennamen bereits wieder angenommen hat, StAZ 53, 284;

Personenstandsgesetz, in: Die Fundstelle, Vorschriftensammlung für die Bayerische Gemeindeverwaltung, Heft 475;

Sind Kinder aus früherer Ehe der geschiedenen Eltern in das Familienbuch aufzunehmen, wenn Vater und Mutter wieder die Ehe miteinander eingehen? StAZ 58, 305;

Vornamensänderung - wenn sich erst später herausstellt, daß bei der Geburtsbeurkundung im Ausland Geschlecht und Vornamen des Kindes unrichtig beurkundet worden sind? StAZ 58, 164;

Zur Auslegung von $\S 15$ b Abs. 1 Satz 3 PStG, StAZ 58, 329;

Zur Neuanlegung des Familienbuches, StAZ 59, 276

Forsthoff: Lehrbuch des Verwaltungsrechts, 1. Bd., Allg. Teil, 7. Aufl., 1958

Frank: Das Recht zur Führung von Adelsnamen, BayStAZ 53, 110

Gätjens: Namensführung der Frau nach öffentlich-rechtlicher Namensänderung des Mannes, StAZ 58, 165

Gaulke: Ein Familienbuch für Heimatvertriebene, DRiZ 58, 173

Geilke: Polnische Adelsnamen, StAZ 59, 189

Gerstein: Zur Änderung des Rufnamens, FamRZ 59, 12

Giese: Neue Rechtsordnung in Ehe und Familie, FamRZ 54, 132

Gieseke : Kann der Standesbeamte stets die Vorlage von Personenstandsurkunden neueren Ausstellungsdatums fordern? StAZ 55, 64

Giffhorn: Der Familienname eines Kindes kann nicht in jedem Falle aus dem Eintrag in dem Geburtenbuch bzw. Geburtsregister mit Sicherheit entnommen werden, StAZ 56, 90; 
Die Befreiung von der Ehemündigkeit hat keine generelle Wirkung, StAZ 60,50 ;

Die Gültigkeitsdauer des Aufgebots, StAZ 55, 244;

Geburt eines Kindes aus totem Mutterleib, StAZ 56, 244;

Kennzeichnung des Rufnamens in den Personenstandsbüchern, Der Hamburger Standesbeamte, 58, 30;

Zu der Frage der Berechtigung von $\S 55$ Abs. 2 EheG, StAZ 60, 211

Gritschneder: Drum prüfe, wer sich ewig bindet, 1954

Grünewald : Erstattungsantrag der Krankenkasse auf Kostenübernahme wegen angeblich falscher Ausstellung der Geburtsbescheinigung, StAZ 57, 80, 323

Grunert: Die Photographie im Standesamt, StAZ 56, 173, 196

Gündisch: Zur Adoption deutscher Kinder in den USA, FamRZ 57, 199

Guggumos: Ausländische Fernadoptionen, NJW 50, 415;

Die Befreiung von der Beibringung des ausländischen Ehefähigkeitszeugnisses, StAZ 54, 2;

Zur Anerkennung der unehelichen Vaterschaft vor rechtskräftiger Anfechtung der Ehelichkeit des Kindes, StAZ 50, 62

Gundrum : Befreiung von der Beibringung des Ehefähigkeitszeugnisses, wenn die Eheschließung nicht vor dem Aufgebotsstandesbeamten stattfindet, StAZ 60,128 ;

Der Familienname der Frau nach dem Gleichberechtigungsgesetz, StAZ 58, 253; Die Eintragung der Staatsangehörigkeit in das Familienbuch, StAZ 58, 310; Familienbuch - Spalte 6, StAZ 59, 228

Gymnich : (Legitimations-?) Randvermerk ohne Legitimation, StAZ 60, 132; Zur Anwendung des $§ 503$ Abs. 1 DA, StAZ 58, 214

Haas: Die Bezeichnung der Anzeigenden im Geburts- und Sterbeeintrag, StAZ 60,295 ;

Die Situation um den Rufnamen, StAZ 59, 266;

Feststellungsbeschlüsse über die Legitimation eines Kindes, StAZ 60, 133

Hamann: Prüfung sowjetzonaler Eheurteile bei Aufgebotsverhandlungen, StAZ 57, 48

Hamel: Glaubens- und Gewissensfreiheit in: Bettermann-Nipperdey-Scheuner, Die Grundrechte, IV/1, 1960

Hansen: Die Vornamen, StAZ 50, 187;

Die Zuständigkeit zur Beurkundung des Sterbefalles bei Wasserleichen,

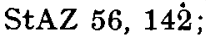

Familienbuch Spalte 9: Unterschiedliche Familiennamen, StAZ 59, 196

Hansen-Fischer: Randvermerke in den Personenstandsbüchern, 1952

Heinisch: Beendigung und Nichtigkeit der Adoption, 1960

Heise: Wiederannahme des früheren Ehenamens durch die geschiedene Ehefrau, StAZ 59, 25

Hen ne: Adoption unter Berücksichtigung des Erleichterungsgesetzes vom 8. 8. 50, damit ein $z u$ erwartendes uneheliches Kind schon bei der Geburt den Namen des Erzeugers erhält - Eheschließung von Adoptivgeschwistern - Name des unehelichen finnischen Kindes, StAZ 52, 45;

Anerkennung ausländischer Eheurteile - Randvermerke zur Heiratsurkunde, StAZ 53, 46;

Bezeichnung des Standesamts im Dienstsiegel - Kreisangabe -, StAZ 56, 68; Die Einwilligung zum Vaterschaftsanerkenntnis eines unter 21 Jahre alten Erzeugers, StAZ 54, 183;

Legitimiert durch rechtskräftigen Beschluß, StAZ 54, 139;

Löschung eines ungültigen Eintrags im Personenstandsbuch, StAZ 52, 224; 
Tod des Kindes vor Ergehen des Legitimationsfeststellungsbeschlusses, StAZ 53, 94;

Zweimalige Eheschließung innerhalb von sieben Tagen an verschiedenen Orten - Löschung des zweiten Heiratseintrags, StAZ 58, 52

Herold: Die Rechtspflichten des Arztes nach dem Personenstandsgesetz, StAZ 60,29

Herre: Bei Legitimation unehelich geborener tschechoslowakischer Kinder im deutschen Geburtsregister genügt die Einhaltung des deutschen formellen Rechts, StAZ 34, 19

Hoegner: Von Beruf und Ausbildung des Standesbeamten, StAZ 53, 169

Hoening: Die Legitimation unehelicher Kinder im deutschen internationalen Privatrecht, Diss. Köln, 1954

Hoffmann: Ausländische Ortsnamen in der standesamtlichen Praxis, StAZ 56,243 ;

Beischreibung eines prozessualen Vaterschaftsanerkenntnisses im Geburtenbuch gemäß § 29 PStG, StAZ 58, 136;

Berufsbezeichnung und Angabe des Wohnortes von Angehörigen der Bundeswehr, StAZ 58, 52;

Besteht eine Lücke im $\S 17$ Abs. 2 PStG und wie könnte sie geschlossen werden? StAZ 52, 43;

Das Gesetz zur Änderung und Ergänzung des Personenstandsgesetzes, StAZ 51, 34;

Der Name des Adoptivkindes einer Frau nach dem Gleichberechtigungsgesetz, StAZ 58, 211;

Die Benachrichtigung in Nachlaßsachen (zu $\S 294$ bis 297 DA), StAZ 51, 185;

Die Haftung für Amtspflichtverletzungen des Standesbeamten, StAZ 53, 170; In welcher Weise kann der Standesbeamte auf die Vornamensgebung Einflu $B$ nehmen? StAZ 51, 62;

Kurzer Grundriß des deutschen Staatsangehörigkeitsrechts, 1960;

Zum Jahreswechsel, StAZ 60, 1

Hubernagel: Die Streitfrage des $\S 1706$ BGB, StAZ 25, 153

Hübschmann: Etwas vom slawischen Adel, StAZ 25, 106;

Vaterschaftsanerkennung seitens eines Russen bzw. eines jetzt staatlosen ehemaligen Russen bei oder nach der Eheschließung mit der Kindesmutter, StAZ 28, 223

Jansen: Freiwillige Gerichtsbarkeit - Handkommentar, 1959

Jonas: Uber die Gültigkeit von Handschuhehen, StAZ 59, 253

Keidel: Aus der Rechtsprechung zur Freiwilligen Gerichtsbarkeit, JZ 58, 16; 59,$438 ; 60,249,312$;

Die neue Gesetzgebung zur Freiwilligen Gerichtsbarkeit, JZ 57, 607;

Gesetz über die Angelegenheiten der Freiwilligen Gerichtsbarkeit, 7. Aufl, 1959

Keimerl : Kann der Standesbeamte die Entgegennahme einer mündlichen Anzeige eines Sterbefalles durch ein Bestattungsunternehmen ablehnen? StAZ 54, 257

Klein: Schreibweise von Vornamen amerikanischer Staatsangehöriger, StAZ 50,284

Knickenberg: Die Bestellung von Standesbeamten bei den Auslandsvertretungen, StAZ 57, 346

Knöchlein: Das Adoptivkind der Frau in namensrechtlicher Hinsicht, StAZ 57,196

Knost: Das „Stammbuch der Familie“ der deutschen Standesbeamten. Seine Entstehung, seine Geschichte und seine heutige Bedeutung, StAZ 58, 11

Koerner: Ausländische Adelsbezeichnungen, StAZ 51, 180 
Kraus: Fehlt das Anerkenntnis durch die Mutter, so ist eine Legitimation nach belgischem Recht nicht möglich; ein dennoch zum Geburtsregister beigeschriebener Legitimationsvermerk muß gelöscht werden, StAZ 58, 165;

Geburtsbeurkundung nach $\S 41 \mathrm{Abs} .1 \mathrm{PStG}$, Anfechtung der Ehelichkeit und Feststellung der Legitimation nach § 31 PStG, StAZ 55, 70

Krauss: Zwangszivilehe und Bonner Grundgesetz, FamRZ 58, 259

Kricke: Das Kind hat die Vornamen erhalten ..., StAZ 56, 227

Krüger: Der Name der Frau nach bürgerlichem Recht, AcP 156, 232

Krüger-Breetzke-Nowack: Gleichberechtigungsgesetz-Kommentar, 1958

Küchenhoff : Besonderer staatlicher Schutz von Ehe und Familie und zeitlicher

Vorrang der standesamtlichen Eheschließung, ZStW 113, 324

Lampe: Voraussetzungen der Adoption, StAZ 58, 85;

Vornamen und ihre Bedeutung, 1949

Laschet: Der Familienname des Adoptierten nach dem Adoptionsvertrag und die Aufnahme des Namens im Randvermerk zum Geburtseintrag, StAZ 60, 267;

Ein abgegebenes Vaterschaftsanerkenntnis schließt eine Namenserteilung (Einbenennung) nach § 1706 BGB nicht aus, StAZ 60, 243;

Namenserteilung (Einbenennung) für ein deutsches Kind, dessen Geburt im Ausland beurkundet wurde ( $\$ 263 \mathrm{DA})$, StAZ 60, 266;

Nur ein kleiner Bindestrich war der Anla $B$ zu einer Berichtigung gemä $B \S 47$ PStG, StAZ 60, 213

Led u c : Ist die personenstandsrechtliche Beurkundung einer Totgeburt notwendig? StAZ 52, 108

Leidag: Zur Verwendung des Wörtchens ,angeblich“ bei der Geburtseintragung, StAZ 49, 155

Lent: Freiwillige Gerichtsbarkeit, 3. Aufl., 1958

Lente: Die Deutsche Dienststelle für die Benachrichtigung der nächsten Angehörigen von Gefallenen der ehemaligen deutschen Wehrmacht, StAZ 59, 19

Lichter: Die Staatsangehörigkeit, 2. Aufl, 1955

Linnartz: Unsere Familiennamen Band I Zehntausend Berufsnamen im Abc, 3. Aufl., 1958;

Band II Aus deutschen und fremden Vornamen im Abc, 3. Aufl., 1958

Lohmann: Ein Kind, das von einer Witwe adoptiert wurde, führt, wenn im Kindesannahmevertrag keine Bestimmung hierüber enthalten ist, nach $\S 1758 \mathrm{a}$ Abs. 3 BGB den Familiennamen, den die Annehmende vor ihrer Verheiratung geführt hat, StAZ 60, 266

Lücke: „Andrea“, StAZ 56, 226;

"Andrea" ein weiblicher Vorname? StAZ 56, 144

Lunczer: Besteht eine Lücke im $\S 17$ Abs. 2 PStG und wie könnte sie geschlossen werden? StAZ 51,$163 ; 52,43$

Mangoldt, von - Klein: Das Bonner Grundgesetz Bd. I, 2. Aufl., 1957

Massfeller: Das gesamte Familienrecht, 1958;

Das polnische Familienrechtsdekret vom 22. Januar 1946 in der Praxis des deutschen Standesbeamten, StAZ 50, 156;

Das unrichtige Vaterschaftsanerkenntnis, StAZ 42, 133;

Der Name des Adoptivkindes einer Frau nach künftigem Recht, StAZ 54, 73;

Deutsches Staatsangehörigkeitsrecht, II. Aufl., 1955;

Deutsch-schweizerische Vereinbarung über den Verzicht auf die Beglaubigung und über den Austausch von Personenstandsurkunden/Zivilstandsurkunden sowie über die Beschaffung von Ehefähigkeitszeugnissen, StAZ 56, 181, 209; Die Anerkennung ausländischer Ehescheidungsurteile in Deutschland, StAZ $37,189,206,224$; 
Die Legitimation eines unehelichen Kindes durch nachfolgende Ehe der EItern, DJ 38, $1381=$ StAZ 39, 37, 54, 80;

Die Namensänderung, JW 38, 337;

Die Novelle zum Personenstandsgesetz, FamRZ 57, 229 ; StAZ 57, 214, 237, 305;

Die Rechtsstellung heimatloser Ausländer im Bundesgebiet, StAZ 51, 130, 155;

Zum Begriff „Wohnort" im Personenstandsgesetz, StAZ 55, 165;

Zum interlokalen Adoptionsrecht, StAZ 55, 25

Massfeller-Reinicke: Das Gleichberechtigungsgesetz - Kommentar, 1958

Maunz: Die verfassungsrechtliche Gewähr von Ehe und Familie (Art. 6 GG), FamRZ 56, 1

Maunz-Dürig: Grundgesetz - Kommentar, 1958

Mehr: Das Zwangsgeldverfahren nach $\S 69$ des Personenstandsgesetzes, StAZ 59,57 ;

Die Ahndung von Ordnungswidrigkeiten nach $\S 68$ der Neufassung des Personenstandsgesetzes, StAZ 58, 169

Meischner: Anfechtung der Ehelichkeit nach dem Tode des Kindes, StAZ 53, 189; Aufgebot und Eheschließung; Zuständigkeit des Standesbeamten, StAZ 59, 305; Berichtigung des Geburtseintrages eines ohne Nachweis als unehelich beurkundeten scheinehelichen Kindes, StAZ 56, 120;

Legitimation eines Kindes nach Namenserteilung, StAZ 55, 46;

Legitimation eines vor Zustellung des Feststellungsbeschlusses verstorbenen Kindes, StAZ 58, 333;

Zeitangabe im Sterbebuch bei Freitod, StAZ 57, 352

Menikheim: Annahme an Kindes Statt eines deutschen unehelichen Kindes durch Ausländer im Ausland, wenn die Vormundschaft für das Kind im Ausland geführt wird, StAZ 60, 273;

Eintragungen im Heiratsbuch und im Familienbuch auf Grund eidesstattlicher Versicherung, StAZ 59, 198

Meth: Die Bestellung eines Standesbeamten in einer kreisangehörigen Gemeinde, StAZ 57, 199

Mörsdorf: „,Der hohe Preis der zivilen Ehe“, FamRZ 54, 123

Motte: Beschaffung von Sterbeurkunden über frühere Angehörige der französischen Fremdenlegion, StAZ 57, 27;

Dürfen auf Grund des Personenstandsgesetzes 1957 noch Fotokopien aus den Personenstandsbüchern ausgefertigt werden? StAZ 59, 124;

Randvermerk über die Legitimation zum Geburtseintrag eines Kindes, dessen Eltern in der sowjetischen Zone die Ehe geschlossen haben, StAZ 59, 80

Mühlbauer: Wiederverehelichung des Ehegatten nach der Todeserklärung oder Feststellung der Todeszeit des anderen Ehegatten - Eintragung eines Randvermerkes über die Auflösung der früheren Ehe, StAZ 53, 111;

Zur Behandlung vor dem Inkrafttreten des PStG 1937 erfolgter Legitimationen, StAZ 57, 350

Müller: Die Schreibweise von ausländischen, insbesondere slawischen Familienund Ortsnamen, StAZ 52, 44;

Legitimations- und Adoptionswirkungen nach BGB, StAZ 56, 294;

Randvermerk nach $\S 30$ PStG bei ,hinkender" Personenstandsveränderung? StAZ 55, 265;

Zur Adoption deutscher Kinder in den USA, NJW 55, 1061

$M u ̈ n c h$, von: Die Grundrechte des Strafgefangenen, JZ 58, 73

Müskens : Berichtigungsverfahren nach $\S 47$ PStG oder Antragstellung nach dem Namensänderungsgesetz bei Änderung des Rufnamens? StAZ 55, 93;

Zuständigkeit für die Beurkundung einer Wasserleiche eines Deutschen, die im Ausland geländet ist, StAZ 54, 45 
Mulle: Der Einfluß des ausländischen Namensrechts auf die Führung der Personenstandsbücher, StAZ 55, 215; 57, 165 ;

Die Bedeutung des Familiennamens der Eltern bzw. der unehelichen Mutter im Geburtseintrag, StAZ 50, 234;

Die Führung der Namensverzeichnisse, StAZ 53, 158;

Die Mitwirkung des Standesbeamten an dem Zustandekommen der Legitimation durch EheschlieBung nach ausländischem Recht, StAZ 56, 202;

Entbindet die Bescheinigung des Aufgebotes den die Eheschließung vornehmenden Standesbeamten jetzt noch von der Pflicht zur Nachprüfung von Ehehindernissen? StAZ 53, 112;

Führung von Adelsbezeichnungen durch unehelich geborene deutsche Staatsangehörige und Berichtigung des $\S 264$ Abs. 1 DA, StAZ 60, 183;

Ist ein doppelter Wohnsitz im Personenstandsbucheintrag zu berücksichtigen und auch sonst zu beachten? StAZ 53, 68;

Ist nach erfolgter Annahme an Kindes Statt ein Vaterschaftsanerkenntnis oder die Feststellung der Legitimation durch nachfolgende Ehe der Eltern am Rande des Geburtseintrages noch zu vermerken? StAZ 53, 189;

Namensführung des Adoptierten nach italienischem Recht, StAZ 52, 190;

Übernahme des Geburtsdatums aus russischen Urkunden, StAZ 50, 189;

Úbersicht über die örtliche Zuständigkeit des Standesbeamten zum Aufgebot und zur Eheschließung, StAZ 55, 66;

Zur Eintragung der Religion in die Personenstandsbücher, StAZ 52, 233;

Zur Notwendigkeit der Weiterführung des Aufgebotsverzeichnisses, StAZ 58,276

Muser: Uber die Beachtung des Ehehindernisses des Ehebruchs bei Scheidungsurteilen aus der sowjetischen Zone, StAZ 57, 49

Neuffer: Adelsbezeichnungen nach russischem Recht, StAZ 55, 92;

Die Konvention der Vereinten Nationen über die Todeserklärung Verschollener, StAZ 55, 201;

Namensänderung durch Hoheitsakt für ein uneheliches Kind und nachfolgende Legitimation durch Eheschließung der Eltern, StAZ 59, 298

Neuffer-Menikheim: Standesamtsführung, 2. Aufl., 1958

Neuhaus: Legitimation und Adoption durch Amerikaner in Deutschland, 9. Beiheft zur DRZ, 49, 7;

Staatliche und kirchliche Eheschließung in rechtsvergleichender Sicht, FamRZ 55, 305

Nied: Eintragung des Familiennamens der Frau in das Familienbuch nach Wiederannahme des Mädchennamens, StAZ 60, 275

Nieden, zur: Änderungen des Personenstandsgesetzes zugunsten von Adoptivkindern und Unehelichen, FamRZ 54, 69;

Die Bedeutung der Geburtsscheine für unehelich Geborene, RdJ 54, 373

Obst: Zur Auslegung des $§ 15$ a Abs. 2 PStG, StAZ 58, 189

Ostendorff: Fortführung des Familienbuches durch den Standesbeamten des Standesamts I in Berlin-West gemä $\$ \S 13$ Abs. 3 PStG, StAZ 59, 105;

Wohnortangaben über die Eltern in den Spalten 4 und 5 des Familienbuches, StAZ 60, 182

Otto: Legitimation eines unehelichen Kindes, dessen Vater Ausländer ist, durch nachfolgende Eheschließung, Rpfleger 50, 9

Palandt: Bürgerliches Gesetzbuch - Kommentar, 19. Aufl., 1960

Peters: Abkürzungen in Personenstandsurkunden, StAZ 58, 305;

Adoption eines deutschen Freiherrn durch eine polnische Freiin, StAZ 59, 333;

Ändert die Befreiung vom Erfordernis der Ehemündigkeit den Status des Befreiten? StAZ 60, 299;

Ausländisches Namensrecht, Rpfleger 53, 109; 
Ausstellung von Heiratsurkunden über die nach dem 31. 12. 1957 geschlossenen Ehen, StAZ 59, 194;

Befreiung von der Beibringung des Ehefähigkeitszeugnisses, wenn die Eheschließung nicht vor dem Aufgebotsstandesbeamten stattfindet, StAZ 59, 248; 60, 128;

Begründet der in einem Aufhebungsurteil festgestellte Ehebruch ein Eheverbot? StAZ 60, 302;

Beischreibung des Legitimationsfeststellungsbeschlusses in den Personenstandsbüchern, StAZ 60, 220;

Beschaffung von Sterbeurkunden über frühere Angehörige der französischen Fremdenlegion, StAZ 57, 234;

Besteht eine Lücke im § 17 Abs. 2 PStG und wie könnte sie geschlossen werden? StAZ 52, 43;

Beurkundung der eidesstaatlichen Versicherung gemäß § 5 (3) PStG durch den zuständigen Standesbeamten, StAZ 60, 190;

Beurkundung eines Schiffssterbefalles, StAZ 58, 279;

Darf ein in Sicherungsverwahrung befindlicher Gewohnheitsverbrecher heiraten? StAZ 60, 276;

Der deutsche Standesbeamte führt seine Personenstandsbücher nach deutschem Recht, StAZ 57, 352;

Der Doktor-Titel, StAZ 52, 91;

Deutsches und italienisches Personenstandsrecht - ein Vergleich, StAZ 59, 169;

Dürfen Vaterschaftsanerkennungen, die bei einem österreichischen Jugendamt beurkundet sind, am Rande eines deutschen Geburtseintrags vermerkt werden? StAZ 56, 252;

Ehelichkeit der Kinder aus hinkenden Ehen, StAZ 59, 277;

Eheschließung an Bord von Seeschiffen, StAZ 54, 115;

Eheverbot der Adoptionsverwandtschaft, StAZ 60, 249;

Einbenennung oder Adoption eines deutschen Kindes durch einen Österreicher, StAZ 53, 238;

Eintragung von ausländischen Eheurteilen in das Familienbuch auf Grund einer eidesstattlichen Erklärung? StAZ 58, 250;

Eintragung von Berufsbezeichnungen in Personenstandsbüchern, StAZ 54, 281 ; Erläuternde Hinweise im Heiratsbuch, die über die in $\S 11$ zugelassenen Angaben hinausgehen, StAZ 59, 165;

Form des Widerrufs der Einwilligung des gesetzlichen Vertreters zur Eheschließung eines Minderjährigen, StAZ 59, 81;

Geburts- und Sterbefälle an der Grenze der Bundesrepublik, StAZ 58, 222;

Gleiche und gleichklingende Namen bei Annahme an Kindes Statt, StAZ 58, 23;

Gleiche Vornamen für Zwillinge, StAZ 60, 191;

Handschuhehe, StAZ 59, 111;

In- und ausländische Adelsbezeichnungen, Rpfleger 53, 223;

In welchen Fällen kann vom Aufgebot befreit werden? StAZ 58, 277; 59, 26;

Ist ,,Kirsten" ein männlicher oder ein weiblicher Vorname? StAZ 60, 247;

Kann der Standesbeamte die Entgegennahme einer mündlichen Anzeige eines

Sterbefalles durch ein Bestattungsunternehmen ablehnen? StAZ 54, 257;

Können die Familienforscher auf die Personenstandsbücher als familiengeschichtliche Quellen verzichten? StAZ 58, 213;

Kollisionsrechtliche Behandlung von Inländern mit zugleich ausländischer Staatsangehörigkeit, StAZ 59, 198;

Legitimation von Kindern, deren Eltern wegen $Z$ weifel an der Gültigkeit ihrer Ehe diese wiederholt haben, StAZ 59, 306;

Löschung von Eheauflösungsvermerken im neuen Heiratsbuch, StAZ 60, 331; Mitteilung der Anerkennung von ausländischen Eheurteilen zum Heiratseintrag, StAZ 55, 91;

Mitteilung der Legitimation eines Ehegatten durch nachfolgende Eheschließung der Eltern zum Heiratseintrag, StAZ 58, 107; 
Mitteilung der Legitimationsfeststellungsbeschlüsse des Vormundschaftsgerichts an die zuständigen Standesämter, Rpfleger 59, 376;

Mitwirkung des gesetzlichen Vertreters eines minderjährigen Anerkennenden bei Beantragung der Beischreibung des Vater- oder Mutterschaftsanerkenntnisses am Rande des Geburtseintrags, StAZ 60, 83;

Namensänderung durch Hoheitsakt für ein uneheliches Kind und nachfolgende Legitimation durch Eheschließung der Eltern, StAZ 59, 82;

Namensführung bei Erstreckung der Adoptionswirkungen auf die Abkömmlinge des Adoptierten, StAZ 60, 225;

Namensführung bei Nichtberücksichtigung des Ehehindernisses der Adoption, StAZ 54, 117;

Neuerungen im Bereich des Personenstandswesens nach Inkrafttreten der Verwaltungsgerichtsordnung, StAZ 60, 130;

Rechtsmittel gegen Ablehnung der Befreiung von der Beibringung des Ehefähigkeitszeugnisses, StAZ 60, 334;

Schreibweise des von einer Frau auf Grund §1355 BGB gebildeten Doppelnamens, StAZ 58, 332;

Ubersicht der in England, den Vereinigten Staaten und Frankreich verliehenen Doktorwüirden, StAZ 52, 234;

Umrechnung von Personenstandsdaten des Julianischen Kalenders, StAZ 39,101 ;

Urkunde über den Ausspruch einer nachträglichen Eheschließung, StAZ 59, 24; Vaterschaftsanerkenntnis für ein vorehelich geborenes Kind nach Heirat der Eltern? StAZ 60, 190;

Wiederannahme des früheren Ehenamens oder Mädchennamens bzw. Untersagung des Mannesnamens, wenn der geschiedenen Frau durch Hoheitsakt erlaubt war, den Mädchennamen dem Mannesnamen hinzuzufügen, StAZ 59, 126 ; Wortlaut a) der in den Personenstandsbüchern beizuschreibenden Randvermerke, b) der in Spalte 8 bis 10 des Familienbuches aufzunehmenden Eintragungen über Personenstandsveränderungen ..., StAZ 59, 193

Peters: Die Gegenwartslage des Staatskirchenrechts, VVDStRL 11, 177

Peterßen : Namensrechtliche Folgen bei einer hinkenden Einbenennung; Führung von zwei Familiennamen, StAZ 58, 275

Pfeiffer: Die Verfassungsbeschwerde in der Praxis, 1959

Piper: Änderung des Drucktextes des Familienbucheintrags, wenn die Brautleute behaupten, nicht miteinander verlobt zu sein? StAZ 57, 25;

Eintragung des Religionsbekenntnisses von Mohammedanern in die Standesregister, StAZ 55, 221

Raape: Die Anerkennung eines ausländischen Ehenichtigkeitsurteils mit besonderer Berücksichtigung des $\S 24$ der 4. EheV., MDR 49, 586;

Die hinkende Scheidung, StAZ 58, 281;

Internationales Privatrecht, 4. Aufl., 1955

Rabel: Aus der Praxis des deutschen internationalen Privatrechts, RabelsZ 32, 310

Reichard: Familienbuch-Eintrag nach Adoption der Ehefrau, StAZ 59, 195;

Vermerke über die Staatsangehörigkeit im Geburtenbuch, StAZ 60, 131

Reichsgerichtsräte-Kommentar : Das Bürgerliche Gesetzbuch ...-Kommentar, herausgegeben von Reichsgerichtsräten und Bundesrichtern, 11. Aufl. im Erscheinen

Reinicke: Rechtswirksame Ehelichkeitserklärung eines unehelich geborenen Kindes durch einen chinesischen Staatsangehörigen, StAZ 27, 125

Rensch: Der adelige Name im deutschen Recht, Diss. Berlin 1931

Rietdorf: Die geschichtliche Entwicklung des Personenstandswesens - Rückblick und Ausblick - (Vortrag), 1957; 


\section{Verzeichnis des Schrifttums}

Die uneheliche Geburt in der Personenstandsurkunde, StAZ 49, 110;

Zum Jahreswechsel, StAZ 59, 1

Rietdorf-Schmidt: Eheerfordernisse und Ehehindernisse, 1960

Rotberg: Gesetz über Ordnungswidrigkeiten - Kommentar, 2. Aufl., 1958

Schätzel : Das Deutsche Staatsangehörigkeitsrecht, 2. Aufl, 1958

Schaumburg: Adoptionsvertrag und Randvermerk hierüber im Geburtseintrag, StAZ 60, 242;

Anordnung des Aufgebots, wenn der Verlobte bei der Bestellung des Aufgebots noch minderjährig ist, bis zur Eheschließung aber das 21. Lebensjahr erreicht hat, StAZ 60, 277;

Beglaubigung der zur Eheschließung erforderlichen Einwilligung, StAZ 60, 277;

Bescheinigung des Aufgebots und standesamtliche Ermächtigung, StAZ 60, 278;

Die Versendung des Familienbuchs, StAZ 59, 269; 60, 19;

Eidesstattliche Versicherungen beim Standesamt, StAZ 59, 77;

Gilt der Sterbebucheintrag, den der Kollege eines benachbarten Standesamts vorgenommen hat? StAZ 60, 334;

Hinweise nach Sterbebeurkundung, StAZ 60, 84;

Hinweismitteilungen zum Familienbuch bzw. Heiratsregister aus Anlaß eines Sterbefalles? StAZ 53, 136;

Randvermerke zum Heiratseintrag und in der Heiratsurkunde, StAZ 60, 248;

Sammelakten beim Standesamt, StAZ 60, 274;

Vermerke über Namensänderungen im Familienbuch, StAZ 60, 276

Zuständigkeit bei Anlegung eines Familienbuches auf Antrag, StAZ 60, 192

Scheurer: Die Namenserteilung nach $\$ 1706$ BGB an ein Schweizer Kind, StAZ 26,74

Scheyhing: Zur Verfassungsmäßigkeit des $\S 67$ des Personenstandsgesetzes, FamRZ 57, 4

Schick: Ist $\S 67$ des Personenstandsgesetzes grundgesetzmäßig? Diss. Tübingen 1957

Schier: Nachtrag eines vergessenen Wissenschaftsvermerkes, StAZ 60, 294; Randvermerk über die Legitimation zum Geburtseintrag eines Kindes, dessen Eltern in der sowjetischen Zone die Ehe geschlossen haben, StAZ 59, 191;

Verlängert sich die einwöchige Anzeigefrist für eine Geburtenanmeldung auch dann, wenn der letzte Anmeldetag kein Sonntag, aber ein Feiertag ist? StAZ 59, 192

Schlegelberger: Gesetz über die Angelegenheiten der Freiwilligen Gerichtsbarkeit, 7. Aufl., 1956

Schmidt: Auseinandersetzungszeugnis bei Heirat eines Adoptivkindes, StAZ 51,21 ;

Beischreibung der Anerkennung der Vaterschaft zum Geburtseintrag des von dem Anerkennenden gezeugten Kindes, StAZ 58, 277;

Beurkundung des Todes eines durch nachfolgende Eheschließung der Eltern legitimierten Kindes, das vor Eintritt der Rechtskraft des LegitimationsFeststellungsbeschlusses verstorben ist, StAZ 50, 165;

Eigentumsrecht an den Personenstandsregistern, StAZ 54, 157;

Der Standesbeamte mu $B$ die Anerkennung der Vaterschaft eines belgischen Staatsangehörigen zu einem unehelichen, von ihm gezeugten Kinde einer deutschen Mutter am Rande des Geburtseintrages beischreiben, StAZ 55, 294; Männlicher Vorname für Mädchen? StAZ 54, 90

Schmitt: Aus der Praxis des Sonderstandesamtes in Arolsen, StAZ 53, 67

Schmitt-Peters: Die Eintragungen in deutsche Personenstandsbücher in Fällen mit Auslandsberührung, 1960

Schnitzerling: Der Name des Adoptivkindes, NJW 60, 566;

Die Fortentwicklung der Adoption seit der Jahrhundertwende, StAZ 60, 57; 
Die vormundschaftsgerichtliche Genehmigung des Adoptionsvertrages und die Zustimmung des gesetzlichen Vertreters, StAZ 60, 169

Schorr: Namenserteilung - Aufnahme der Erklärung des Ehemannes in die Heiratsurkunde bei Nichtvorliegen der Einwilligung des Kindes bzw. seines Vormunds, StAZ 58, 23;

Namenserteilung durch Ausländer bzw. Staatenlose, StAZ 50, 70

Schramm: Lebendgeburt, Zwitter und Mißgeburt — juristisch-medizinische Grenzprobleme bei Eintragungen im Geburtenbuch, StAZ 40, 91

Schrembs: Vaterschaftsanerkenntnis, BayStAZ 53, 55;

Zur Beurkundung nach $\S 41$ PStG, StAZ 52, 259

Schröer: Die Ehemündigkeit, StAZ 52, 241;

Die familienrechtliche Gesetzgebung der sowjetischen Zone in ihrer Auswirkung in der Bundesrepublik, StAZ 54, 75

Schütte: Zuständigkeit zur Entgegennahme von Erklärungen über Namenserteilung bei unehelichen Kindern, die in der sowjetischen Zone geboren sind, und gesetzliche Vertretung dieser Kinder, StAZ 58, 277

Schultheis: Das Auseinandersetzungszeugnis und die Aufgebotsverhandlung, StAZ 58, 157;

Geburtsurkunde und Eintrag in den Spalten 2, 5 und 10 des Familienbuches einer Frau, die unehelich geboren ist und deren Familienname sich durch Adoption und behördliche Namensänderung geändert hat, StAZ 59, 166

Schwoerer: Die Feststellung der Legitimation unehelicher Kinder nach $\S 31$ PStG, § 22 AVO, DFG 39, 185;

Feststellung der Legitimation unehelicher Kinder nach § 31 PStG unter Anwendung des Badischen Landrechts, StAZ 40, 64;

Feststellung der Legitimation unehelicher Kinder nach $\S 31$ PStG unter Anwendung des Code civil, DFG 40, 2;

Kann das Vormundschaftsgericht seinen nach $\S 31$ PStG erlassenen Legitimationsfeststellungsbeschlu $B$ wieder aufheben, wenn sich herausstellt, da $B$ dieser Beschluß sachlich unrichtig ist? DFG 42, 95;

Legitimation unehelicher Kinder durch nachfolgende Ehe und Anfechtung des Staatsanwalts, DFG 40, 165;

Wird das uneheliche Kind einer deutschen Mutter durch nachfolgende Ehe legitimiert, wenn ein Franzose die Mutter heiratet und dabei vor dem französischen Standesbeamten das Kind anerkennt, obwohl er nicht der Vater ist? StAZ 53, 145

Seeler: Die Staatsangehörigkeit der Volksdeutschen, 1960

Sievers: Fremde Adelsnamen in der Schweiz, Deutschland und Österreich, StAZ 31, 295, 313

Soergel : Bürgerliches Gesetzbuch nebst Einführungsgesetz, 9. Aufl. im Erscheinen

Spanner: Aufhebung des $\S 67$ Personenstandsgesetz durch den österr. Verfassungsgerichtshof, DÖV 56, 292

Sperling, Die Wiedergabe russischer Namen in lateinischer Schrift (§ 112 DA), StAZ 53, 255

Staudinger, von: Kommentar zum Bürgerlichen Gesetzbuch und dem Einführungsgesetz, 11. Aufl. im Erscheinen

Stelter: Sind Zweitbücher wirklich unentbehrlich? StAZ 50, 65

Stölzel: Die Rechtsstellung des Standesbeamten, StAZ 36, 118; Legitimation durch einen Russen, StAZ 24, 280;

Namensrecht, 1939

Stölzel-Peters: Die rechtliche Stellung des Standesbeamten, 1944

Stoldt: Doppelte Prüfung von Kindesannahmeverträgen? SchlHA 56, 345

Strunck: Antrag auf Anlegung eines Familienbuches gemä $B$ § 15a PStG, StAZ 58,274 


\section{Verzeichnis des Schrifttums}

Thias: Austritt aus einer ausländischen Religionsgemeinschaft, StAZ 40, 223;

Bedeutung der Rechtskraft des Legitimationsfeststellungsbeschlusses, StAZ 55,272 ;

Die Bedeutung der Anerkennung der Vaterschaft zu unehelichen Kindern, besonders der Anerkennung durch Ausländer, StAZ 24, 145;

Ehelich oder unehelich wegen Nichtigkeit der Ehe der Eltern? StAZ 56, 150; Genügt die Mitteilung eines ausländischen Standesbeamten über die Anerkennung eines unehelichen Kindes als Grundlage für die Beischreibung zum Geburtseintrag, StAZ 53, 140;

Probleme bei Ortsangaben - veränderte Ortsnamen und Standesamtsbezirke, StAZ 53, 160;

,Scheineheliches Kind ist schon als unehelich und später legitimiert beurkundet", StAZ 56, 48;

Seltener Fall des Vaterschaftsanerkenntnisses durch Minderjährige, StAZ 55,146 ;

Zweifel vor der Eintragung eines Adoptionsvermerkes, StAZ 57, 53

Thomsen: Das Recht auf Benutzung der Personenstandsbücher, StAZ 59, 141;

Die Staatsangehörigkeit in der Standesamts-Praxis, StAZ 59, 281;

Zur Änderung des Rufnamens, FamRZ 59, 92

Völker: Das gerichtliche Verfahren in Personenstandssachen, StAZ 52, 73;

Randvermerke und Hinweise nach neuem Verschollenheitsrecht, StAZ 51, 228

Voß: Zur Versagung der Anerkennung sowjetzonaler Scheidungsurteile analog $\S 328$ I Nr. 1 i. Verbdg. m. §606a ZPO n.F., FamRZ 59, 189

Wagner: Abschluß der Personenstandsbücher und Namensverzeichnisse, StAZ 49,32 ;

Darf die Annahme an Kindes Statt beim Aufgebot verschwiegen werden? (§ $153 \mathrm{DA})$, StAZ 54, 116;

Darf die Mutter eines unehelichen Kindes im Geburtseintrag als „ledig" bezeichnet werden, StAZ 53, 257;

Das fehlende Auseinandersetzungszeugnis als Ehehindernis, StAZ 55, 163;

Das scharfe ,"s", StAZ 59, 321;

Eidesstattliche Versicherungen, StAZ 53, 181;

Familienstand des Verstorbenen im Sterbebuch, StAZ 55, 116;

Geburtsanzeige eines Ausländers, StAZ 37, 49;

Personenstandsbücher aus älterer Zeit, StAZ 57, 227;

Standesamt und Aufsicht, StAZ 49, 132, 151, 216;

Standesbeamter, Stellvertreter und ihre Verantwortung, StAZ 55, 87, 238, 291 ;

Sterbefallanzeigen an die Reichskartei für Testamente, StAZ 55, 273

Waldmann: $\mathrm{Zu}$ den gerichtlichen Entscheidungen nach dem Personenstandsgesetz, DFG 43, 63

Wickenhäuser: Das Berichtigungsverfahren nach dem neuen Personenstandsgesetz, StAZ 59, 327

Wiese: Abkürzungen und Übertragungen, StAZ 60, 243

Wutz: Die neuen amtlichen Vordrucke, StAZ 58, 46

Zacher: Adoption eines deutschen ehelichen Kindes durch ein französisches Ehepaar, StAZ 54, 260;

Die Aufbewahrung der Zweitbücher für das laufende Jahr, StAZ 60, 79

Zschacke: Über die Nachweisung und Geltendmachung der Unehelichkeit seitens eines durch nachfolgende Eheschließung Legitimierten, JW 37, 2499. 
A.

Recht des Personenstandes

in der

Bundesrepublik Deutschland 
